Viviana Lenis Ballesteros ${ }^{1}$

Yolanda Lucía López Arango"

Yicenia Milena Cuadros Urrego
Programa de Maestría en Epidemiologia. Universidad de Antioquia. Medellín, Colombia

Programa de Maestría en Salud Pública. Universidad de Antioquia. Medellín, Colombia

Correspondência | Correspondence: Viviana Lenis Ballesteros Carrera 76108 - 11, Florencia 05001000 Medellín, Antioquia, Colômbia E-mail: vileba15@gmail.com

Recibido: $13 / 6 / 2011$

Aprobado: 16/4/2012

Artículo disponible en español y ingles en: www.scielo.br/rsp

\section{Condiciones de salud y de trabajo informal en recuperadores ambientales del área rural de Medellín, Colombia, 2008}

\section{Health and informal work conditions among recyclers in the rural area of Medellin, Colombia, 2008}

\section{RESUMEN}

OBJETIVO: Caracterizar las condiciones de trabajo, salud y riesgos ocupacionales de recuperadores ambientales del área rural de Medellín.

MÉTODOS: Estudio transversal con cien recuperadores ambientales informales en cinco corregimientos de Medellín en 2008. La fuente de información fue primaria: una encuesta estructurada sobre condiciones de trabajo, salud, riesgos ocupacionales y morbilidad sentida, que permitió caracterizar los riesgos de la población mediante estadística descriptiva y un acercamiento a las relaciones y asociaciones de los riesgos con la morbilidad en la población.

RESULTADOS: Ciento cuatro personas ejercían el reciclaje, y 100 de estos se incluyeron en el estudio: 34 personas agremiadas en organizaciones de base comunitaria y 66 personas que ejercían la labor independientemente. La afiliación a seguridad social, suplencia de necesidades básicas, satisfacción en la labor, recibir capacitaciones y suministro periódico de elementos para su trabajo presentaron diferencias según grupo $(\mathrm{p}<0,05)$. Los riesgos biológicos, relacionados con postura corporal inadecuada, riesgos físicos y químicos, tuvieran frecuencia particular de exposición según grupo. El conocimiento y uso de las medidas de protección para los riesgos de la labor difirieron estadísticamente entre los grupos $(\mathrm{p}<0,05)$.

CONCLUSIONES: Los recuperadores son potencial apoyo para la gestión integral de residuos en áreas rurales de Medellín, aunque su carácter informal los expone a condiciones de trabajo que repercuten directamente en su salud y la de sus familias. El reconocimiento social del reciclaje va de la mano con la mejora de las condiciones laborales de las personas dedicadas a esta actividad. Los recuperadores ambientales, aun agremiados, tienen condiciones laborales y de salud precarias. La trasformación de estas condiciones debería ser una de las prioridades del Estado.

DESCRIPTORES: Trabajadores Rurales. Segregadores de Residuos Sólidos. Reciclaje. Riesgos Laborales. Condiciones de Trabajo. Salud Laboral. Estudios Transversales. 


\begin{abstract}
OBJECTIVE: To characterize the work, health and occupational risk conditions of recyclers from the rural area.

METHODS: Cross-sectional study, with the participation of one hundred informal recyclers in five corregimientos (small administrative units) of Medellin in 2008. The source of information was primary: a structured survey on work, health and occupational risk conditions and also on perceived morbidity, which enabled to characterize the population's risks through descriptive statistics and approach the relationships and associations of the risks with the morbidity in the population.
\end{abstract}

RESULTS: One hundred and four people were engaged in recycling, and 100 of them were included in the study: 34 people belonged to community-based organizations and 66 people worked independently. Social security affiliation, fulfillment of basic needs, work satisfaction, access to training activities and periodic provision of work supplies presented differences according to group $(\mathrm{p}<0.05)$. The biological risks, associated with inadequate body postures, and physical and chemical risks had a particular frequency of exposure according to group. The knowledge and use of safety measures for occupational risks differed statistically among the analyzed groups $(\mathrm{p}<0.05)$.

CONCLUSIONS: Recyclers are a potential support to integral waste management in rural areas of Medellin, although the informal nature of their work exposes them to working conditions that directly affect their health and the health of their families. The social recognition of recycling goes hand in hand with the improvement in the working conditions of the people engaged in this activity. The environmental recycling workers, even if belonging to a union, have precarious work and health conditions. The transformation of these conditions should be one of the State's priorities.

\section{DESCRIPTORS: Rural Workers. Solid Waste Segregators. Recycling. Occupational Risks. Working Conditions. Occupational Health. Cross- Sectional Studies.}

\section{INTRODUCCIÓN}

La creciente urbanización mundial, derivada del aumento acelerado de la población y la industrialización, involucra la generación cada vez mayor de residuos sólidos. Su manejo es una temática de interés, dadas sus implicaciones ambientales y sanitarias. ${ }^{11} \mathrm{El}$ problema de la basura no es exclusivo de ciudades grandes; también ha tocado las pequeñas poblaciones. ${ }^{a}$

El reciclaje ha tomado relevancia en las últimas décadas debido a que se ha convertido en una estrategia de disminución de residuos con bajo costo. Es un "nicho de mercado", donde las ventajas se visualizan en la economía de las industrias, con la consecuente disminución de costos en materia prima, y se ha convertido en una opción de ingresos en comunidades de escasos recursos y falta de oportunidades estables de empleo. ${ }^{8}$

La generación diaria de residuos se estima en 28.800 toneladas en Colombia, $45 \%$ aproximadamente con potencial de reutilización, pero solo el $7 \%$ de esta cantidad es recuperado, a pesar de ser fuente de ingresos de una población importante. Las organizaciones de reciclaje registraron 20.000 familias dedicadas a esta labor en 2006. ${ }^{\mathrm{b}}$ En el área metropolitana del Valle de Aburrá de Antioquia se contaba con 3.190 recuperadores ambientales en ese año, más de 2.000 en la ciudad de Medellín.

\footnotetext{
a Organización Panamericana de la Salud. Centro Panamericano de Ingeniería Sanitaria y Ciencias del Ambiente. Seminario internacional sobre rellenos sanitarios y reciclaje. Lima; 1989.

${ }^{\mathrm{b}}$ Ministerio de Ambiente, Vivienda y Desarrollo Territorial. Por el desarrollo sostenible del reciclaje: propuesta inicial. Bogotá; 2006. c Sepúlveda-Villada LA. Evaluación económica, social y ambiental de la recuperación de residuos aprovechables con la participación del reciclador informal. Estudio de caso: Barrios Floresta y Santa Lucia de Medellín. Medellín: Universidad Nacional de Colombia; 2005 [citado 2012 ene 7]. Disponible en: http://www.resol.com.br/textos/EVALUACION\%20FINAL\%20ECONOMICA\%20SOCIAL\%20Y\%20 AMBIENTAL\%20DEL\%20RECICLAJE.pdf
} 
El proceso de reciclaje tiene tres grandes etapas: recuperación, transformación y comercialización de los residuos reutilizables. Los recuperadores se relacionan en la primera etapa; son ellos los encargados de buscar y seleccionar el material que será posteriormente reutilizado en otras industrias, constituyéndose en actores sociales importantes en el manejo y gestión de los residuos sólidos. Como personal involucrado en el contacto con la basura y bajo condiciones de trabajo de estas personas no les permiten el desarrollo pleno de sus habilidades, se exponen a riesgos para la salud y su integridad física, propios de su actividad, ${ }^{10,14}$ y que no les permite contar con las garantías de seguridad social establecidas por la ley.

Esto lleva a la precariedad en la vida de estas personas y a que la mirada de la sociedad niegue su condición de iguales. ${ }^{2}$

Los riesgos inherentes a la labor de reciclaje, de carácter biológico, físico, químico, ergonómico, público y psicosocial, se potencian ante la ausencia de mecanismos efectivos para el acceso a servicios de salud y de asistencia social. ${ }^{8}$

Las características de la labor del reciclaje, entre ellas, la generación de bajos ingresos, pocas oportunidades de progreso debido al bajo perfil de la profesión por su poca exigencia de conocimiento y educación, la inestabilidad en el trabajo, escasa protección en seguridad social, condiciones precarias de trabajo y la casi nula protección frente a riesgos ocupacionales, conlleva a que esta labor se ubique dentro de la economía informal del país. ${ }^{2}$

En los últimos años, se han realizado diferentes esfuerzos para apoyar la conformación de cooperativas $\mathrm{u}$ organizaciones sociales dirigidas a promover la organización de estos colectivos para la capacitación empresarial, el fortalecimiento de su labor y el mejoramiento de sus condiciones de vida. ${ }^{\mathrm{d}}$

Las autoridades ambientales han promovido diferentes procesos para inclusión de los recuperadores ambientales en la gestión social integral de los residuos sólidos, involucrando, desde el 2004, a los recuperadores habitantes de las áreas rurales de la ciudad de Medellín.

Este trabajo tuvo como objetivo caracterizar las condiciones de trabajo, de salud y de riesgos ocupacionales de recuperadores ambientales del área rural de Medellín.

\section{MÉTODOS}

Estudio exploratorio transversal en cinco corregimientos del área rural de Medellín: Santa Elena, San
Antonio de Prado, San Sebastián de Palmitas, AltaVista y San Cristóbal en 2008.

La población de recuperadores ambientales en la zona de interés se identificó mediante un censo que involucró recorridos en las calles de las localidades en los días de recolección de basura.

La recolección de la información se realizó a partir de encuesta estructurada que abordaba temáticas de condiciones de trabajo, de salud, de riesgos ocupacionales y de morbilidad sentida por la población.

Se censaron 104 personas dedicadas a la labor de recuperación de residuos sólidos en los corregimientos, de los cuales, 96,4\% participaron en el estudio mediante consentimiento informado escrito.

Para el análisis de los datos, se usó el programa SPSS versión 11, obteniéndose frecuencias relativas, proporciones, razones de prevalencia (RP), intervalo de confianza (IC95\%) y valor $\mathrm{p}(<0,05)$ para identificar diferencias estadísticas entre los dos grupos de recuperadores.

Cada recuperador manifestó su interés en participar de la investigación mediante consentimiento informado por escrito. La investigación fue catalogada como una investigación sin riesgo, según la Resolución 8.430 de 1993, del Ministerio de Salud de Colombia.

\section{RESULTADOS}

Treinta y cuatro personas ejercían la labor dentro de una organización social integrada al centro de aprovechamiento de residuos de los corregimientos y 66 ejercían la labor independientemente (Tabla 1).

Predominaron hombres dedicados a la labor en ambos grupos. En el grupo de los recuperadores agremiados a la organización social del centro de aprovechamiento

Tabla 1. Población de recuperadores según corregimiento. Área rural de Medellín, Colombia, 2008.

\begin{tabular}{lcccc}
\hline Corregimiento & $\begin{array}{c}\text { Recuperadores } \\
\text { agremiados }\end{array}$ & \multicolumn{2}{c}{$\begin{array}{c}\text { Recuperadores } \\
\text { independientes } \\
\text { no agremiados }\end{array}$} \\
& $\mathrm{n}$ & $\%$ & $\mathrm{n}$ & $\%$ \\
\hline San Antonio de Prado & 11 & 32,4 & 32 & 48,5 \\
San Cristóbal & 7 & 20,6 & 11 & 16,7 \\
Altavista & 6 & 17,6 & 12 & 18,2 \\
Santa Elena & 6 & 17,6 & 10 & 15,2 \\
Palmitas & 4 & 11,8 & 1 & 1,5 \\
\hline Total & 34 & 100,0 & 66 & 100,0 \\
\hline
\end{tabular}

d Ramírez-Guerrero J. Capacitación laboral para el sector informal en Colombia. Ginebra: Oficina Internacional del Trabajo; 2002 [citado 2012 ene 7]. Disponible en: http://www.oei.es/etp/capacitacion_laboral_sector_informal_colombia_guerrero.pdf 
Tabla 2. Características de la población de recuperadores ambientales de los cinco corregimientos. Área rural de Medellín, Colombia, 2008.

\begin{tabular}{lcc}
\hline $\begin{array}{l}\text { Características de la } \\
\text { población }\end{array}$ & $\begin{array}{c}\text { Agremiados } \\
(\mathrm{n}=34)\end{array}$ & $\begin{array}{c}\text { No agremiados } \\
(\mathrm{n}=66)\end{array}$ \\
\hline Edad & 32 & 52 \\
$\quad$ Mediana & 57 & 82 \\
$\quad$ Máximo & 18 & 15 \\
$\quad$ Mínimo & & \\
Sexo & $64,7 \%(22)$ & $68,2 \%(45)$ \\
Hombre & $35,3 \%(12)$ & $31,8 \%(21)$ \\
Mujer & & \\
Estado civil & $52,9 \%(18)$ & $37,9 \%(25)$ \\
Soltero & $17,6 \%(6)$ & $42,4 \%(28)$ \\
Casado & $17,6 \%(6)$ & $9,1 \%(6)$ \\
Unión libre & 0 & $3,0 \%(2)$ \\
Viudo & $11,9 \%(4)$ & $7,6 \%(5)$ \\
Separado & $2,9 \%(1)$ & $6,1 \%(4)$ \\
Nivel de estudio determinado por ciclo completo \\
Ninguno & $11,8 \%(4)$ & $54,5 \%(36)$ \\
Primaria & $55,9 \%(19)$ & $36,4 \%(24)$ \\
Secundaria & $29,4 \%(10)$ & $9,1 \%(6)$ \\
Técnico & $2,9 \%(1)$ & $0 \%(0)$ \\
Estrato socioeconómico & & $34,8 \%(23)$ \\
1 & $11,8 \%(4)$ & $59,1 \%(39)$ \\
2 & $85,3 \%(29)$ & \\
\hline & & \\
& &
\end{tabular}

se encontró mayor frecuencia de personas en edades productivas y nivel educativo mayor, en comparación con los no agremiados, quienes tenían mayor frecuencia de personas con primaria y secundaria completa e incluso con formación técnica (Tabla 2).

Ambos grupos, agremiados y no agremiados, resaltaron que su labor no solo era una forma de sustento económico y de ser útiles en la economía, sino que también aportaba a la sociedad, en tanto contribuían directamente al mantenimiento de un ambiente más limpio (percepción mayor del $40 \%$ en recuperadores no agremiados y mayor del $59 \%$ en los agremiados).

Las jornadas laborales (en horas por día y en días por semana), además de la necesidad de complementar con otros oficios, fue similar en ambos grupos; la autonomía en el trabajo, la aplicación de sus conocimientos en la labor y el descanso durante la semana no tuvieron diferencias estadísticamente significativas entre los grupos (Tabla 3).

La suplencia de las necesidades básicas por los ingresos derivados de la labor, la satisfacción en la labor, trabajar en compañía, hacer ejercicios de relajación, recibir capacitaciones y tener dotaciones periódicas y suministro de elementos para su trabajo, presentaron diferencias estadísticas significativas a favor de los recuperadores agremiados a los centros de aprovechamiento (Tabla 3).

Existieron diferencias con relación a la distancia recorrida para la recolección del material reciclable, con

Tabla 3. Condiciones de trabajo de los recuperadores ambientales. Área rural de Medellín, Colombia, 2008.

\begin{tabular}{|c|c|c|c|c|c|c|c|c|c|c|c|}
\hline \multirow{3}{*}{ Condiciones de trabajo } & \multicolumn{4}{|c|}{$\begin{array}{l}\text { Recuperador } \\
\text { agremiado }\end{array}$} & \multicolumn{4}{|c|}{$\begin{array}{l}\text { Recuperador no } \\
\text { agremiado }\end{array}$} & \multirow{3}{*}{ OR } & \multirow{3}{*}{ IC95\% } & \multirow{3}{*}{$\mathrm{p}^{\mathrm{a}}$} \\
\hline & \multicolumn{2}{|c|}{$\mathrm{Si}$} & \multicolumn{2}{|c|}{ No } & \multicolumn{2}{|c|}{ Si } & \multicolumn{2}{|c|}{ No } & & & \\
\hline & $\%$ & $\mathrm{n}$ & $\%$ & $\mathrm{n}$ & $\%$ & $\mathrm{n}$ & $\%$ & $\mathrm{n}$ & & & \\
\hline Jornada laboral menor o igual a 8 horas día & 51,5 & 17 & 48,5 & 16 & 63,6 & 42 & 36,4 & 24 & 1,41 & 0,$66 ; 3,0$ & 0,36 \\
\hline Días laborados semana menor o igual a 5 & 81,8 & 27 & 18,2 & 6 & 85,5 & 53 & 14,4 & 9 & 0,76 & 0,$24 ; 2,37$ & 0,64 \\
\hline El ingreso suple las necesidades & 55,9 & 19 & 44,1 & 15 & 30,3 & 20 & 62,1 & 41 & 2,59 & 1,$09 ; 6,15$ & 0,02 \\
\hline Oficio alterno & 32,4 & 11 & 55,9 & 19 & 42,4 & 28 & 48,5 & 32 & 1,5 & 0,$61 ; 3,71$ & 0,36 \\
\hline Trabaja en compañía & 100 & 34 & 0 & 0 & 54,5 & 36 & 43,9 & 29 & b & & 0 \\
\hline Distancia recorrida menor de 500 metros & 55,2 & 16 & 44,8 & 13 & 78,8 & 41 & 21,2 & 11 & 0.33 & 0,$12 ; 0,88$ & 0,02 \\
\hline Satisfacción ejerciendo la labor & 97,1 & 33 & 0 & 0 & 63,6 & 42 & 24,2 & 16 & b & & 0,0009 \\
\hline Autonomía para desarrollar la labor & 94,1 & 32 & 5,9 & 2 & 93,9 & 62 & 3 & 2 & 0,51 & 0,$06 ; 3,83$ & 0,43 \\
\hline Aplicación de conocimientos en la labor & 97,1 & 33 & 2,9 & 1 & 87,9 & 58 & 10,6 & 7 & 3,98 & 0,$47 ; 33,7$ & 0,16 \\
\hline Descanso durante la semana & 85,3 & 29 & 14,7 & 5 & 98,4 & 61 & 7,6 & 5 & 0,47 & 0,$12 ; 1,77$ & 0,26 \\
\hline Ejercicios de relajación & 64,7 & 22 & 35,3 & 12 & 9,1 & 6 & 42,4 & 28 & 8,55 & 2,$76 ; 26,42$ & 0,0001 \\
\hline Suministro de dotaciones alguna vez & 100 & 34 & 0 & 0 & 27,3 & 18 & 69,7 & 46 & b & & 0 \\
\hline Dotaciones trimestrales de los suministros & 64,7 & 22 & 22,2 & 8 & 4,5 & 3 & 95,5 & 63 & 57,7 & 14,$05 ; 237,9$ & 0 \\
\hline Capacitaciones recibidas & 94,1 & 32 & 2,9 & 1 & 25,8 & 17 & 72,7 & 48 & 90,4 & 11,$44 ; 713$ & 0,000 \\
\hline
\end{tabular}

a Significancia estadística con valores $\mathrm{p}<0,05$

b No se calcula dado que una de las frecuencias observadas es 0 
Tabla 4. Factores de riesgo derivados de la labor para los recuperadores ambientales. Área rural de Medellín, Colombia, 2008.

\begin{tabular}{|c|c|c|c|c|c|c|c|c|c|c|c|}
\hline \multirow{3}{*}{ Factor de riesgo } & \multicolumn{4}{|c|}{ Recuperador agremiado } & \multicolumn{4}{|c|}{ Recuperador no agremiado } & \multirow{3}{*}{ OR } & \multirow{3}{*}{ IC95\% } & \multirow{3}{*}{$\mathrm{p}^{\mathrm{a}}$} \\
\hline & \multicolumn{2}{|c|}{$\mathrm{Si}$} & \multicolumn{2}{|c|}{ No } & \multicolumn{2}{|c|}{$\mathrm{Si}$} & \multicolumn{2}{|c|}{ No } & & & \\
\hline & $\%$ & $\mathrm{n}$ & $\%$ & $\mathrm{n}$ & $\%$ & $\mathrm{n}$ & $\%$ & $\mathrm{n}$ & & & \\
\hline \multicolumn{12}{|l|}{ Biológico } \\
\hline Material en descomposición & 88,2 & 30 & 11,8 & 4 & 97,0 & 64 & 3,0 & 2 & 4,2 & 0,$74 ; 24,5$ & 0,1 \\
\hline $\begin{array}{l}\text { Material cortante } y / o \\
\text { contaminado con fluidos }\end{array}$ & 94,1 & 32 & 5,9 & 2 & 100,0 & 66 & 0 & 0 & b & b & 0,04 \\
\hline \multicolumn{12}{|l|}{ Físico } \\
\hline Trabajo a la intemperie & 100,0 & 34 & 0 & 0 & 95,5 & 63 & 4,5 & 3 & b & b & b \\
\hline Trabajo en sitios cubiertos & 100,0 & 34 & 0 & 0 & 43,9 & 29 & 56,1 & 37 & b & b & b \\
\hline $\begin{array}{l}\text { Iluminación suficiente en sitios } \\
\text { cubiertos }\end{array}$ & 64,7 & 22 & 26,5 & 9 & 36,4 & 24 & 6,1 & 4 & 2,28 & 0,$65 ; 8,0$ & 0,14 \\
\hline Trabajo en horas nocturnas & 73,5 & 25 & 8,8 & 3 & 78,8 & 52 & 12,1 & 8 & 0,84 & 0,$22 ; 3,21$ & 0,51 \\
\hline $\begin{array}{l}\text { Iluminación en trabajo } \\
\text { nocturno }\end{array}$ & 20,0 & 5 & 80,0 & 20 & 17,3 & 9 & 82,7 & 43 & 0,81 & 0,$25 ; 2,63$ & 0,5 \\
\hline Trabajo en zonas con polvo & 64,7 & 22 & 35,3 & 12 & 60,6 & 40 & 31,8 & 21 & 1,04 & 0,$43 ; 2,49$ & 0,91 \\
\hline Vibraciones & 64,7 & 22 & 35,3 & 12 & 33,3 & 22 & 66,7 & 44 & 0,28 & 0,$11 ; 0,66$ & 0,003 \\
\hline Ruido & 91,2 & 31 & 8,8 & 3 & 83,3 & 55 & 16,7 & 11 & 0,53 & 0,$15 ; 1,91$ & 0,22 \\
\hline Frío & 79,4 & 27 & 20,6 & 7 & 77,2 & 51 & 21,2 & 14 & 0,96 & 0,$35 ; 2,62$ & 0,95 \\
\hline Calor & 79,4 & 27 & 20,6 & 7 & 87,9 & 58 & 12,1 & 8 & 1,8 & 0,$63 ; 5,53$ & 0,24 \\
\hline Cambios temperatura & 58,8 & 20 & 41,2 & 14 & 83,3 & 55 & 16,7 & 11 & 3,4 & 1,$35 ; 8,6$ & 0,007 \\
\hline Fuentes energía & 79,4 & 7 & 20,6 & 27 & 12,1 & 8 & 81,8 & 54 & 0,57 & 0,$19 ; 1,69$ & 0,3 \\
\hline \multicolumn{12}{|c|}{ Relacionado con posturas corporales inadecuadas } \\
\hline Levantamiento de cargas pesadas & 79,4 & 27 & 20,6 & 7 & 69,7 & 46 & 30,3 & 20 & 0,61 & 0,$23 ; 1,61$ & 0,32 \\
\hline Halar cargas pesadas & 64,7 & 22 & 35,3 & 12 & 62,1 & 41 & 37,9 & 25 & 0,9 & 0,$38 ; 2,11$ & 0,81 \\
\hline Posición incómoda & 70,6 & 24 & 29,4 & 10 & 59,1 & 39 & 60,9 & 27 & 0,61 & 0,$25 ; 1,47$ & 0,27 \\
\hline Movimientos repetitivos & 94,1 & 32 & 2,9 & 1 & 81,8 & 54 & 18,2 & 12 & 0,2 & 0,$03 ; 1,15$ & 0,02 \\
\hline Estiramientos & 55,9 & 19 & 44,1 & 15 & 54,5 & 36 & 45,5 & 30 & 0,95 & 0,$41 ; 2,16$ & 0,9 \\
\hline \multicolumn{12}{|l|}{ Químico } \\
\hline Aspiración de gases o vapores & 79,4 & 27 & 20,6 & 7 & 72,7 & 48 & 27,3 & 18 & 0,71 & 0,$27 ; 1,88$ & 0,49 \\
\hline
\end{tabular}

a Significancia estadística con valores $\mathrm{p}<0,05$

b No se calcula dado que una de las frecuencias observadas es 0

Los porcentajes fueron calculados teniendo como denominador las poblaciones captadas de recuperadores agremiados $(n=34)$ y recuperadores no agremiados $(n=66)$.

significancia estadística a favor de los recuperadores no agremiados, quienes recorren en su mayoría distancias menores a $500 \mathrm{~m}$ (Tabla 3); los recuperadores agremiados poseían mayor cobertura de las zonas dada la disponibilidad de vehículo en el recorrido, así como, mayores y mejores medios e instrumentos de trabajo.

Ejercer el reciclaje, siendo parte de una organización social empresarial, permitió que los recuperadores tuvieran garantías en seguridad social establecidas por la Ley 100 de 1993, como salud, pensión y afiliación a aseguradoras de riesgos profesionales; el 57\% de los recuperadores no agremiados contaban con vinculación al sistema de salud, la mayoría a partir del régimen subsidiado garantizado por el Estado. Diez recuperadores no agremiados contaban con todas las garantías en seguridad social derivadas de los oficios alternos que ejercían dichas personas. Las diferencias en seguridad social fueron estadísticamente significativas en ambos grupos a favor de los recuperadores agremiados a los centros de aprovechamiento.

Ambos grupos se enfrentaban a factores de riesgo del orden biológico, relacionados con posturas corporales inadecuadas, riesgos físico y químico, inherentes a su labor. Se presentaron diferencias significativas con relación a la mayor frecuencia de exposición de los recuperadores no agremiados por el contacto con material cortante $\mathrm{y} / \mathrm{o}$ contaminado con fluidos y a los cambios bruscos de temperatura $(\mathrm{p}<0,05)$. Los recuperadores agremiados se exponían con mayor frecuencia a vibraciones y los movimientos repetitivos $(\mathrm{p}<0,05)$, 
Tabla 5. Eventos de morbilidad sentida en el último mes por los recuperadores ambientales. Área rural de Medellín, Colombia, 2008.

\begin{tabular}{|c|c|c|c|c|c|c|c|c|c|c|c|}
\hline \multirow{3}{*}{ Afección } & \multicolumn{4}{|c|}{ Recuperador agremiado } & \multicolumn{4}{|c|}{ Recuperador no agremiado } & \multirow{3}{*}{ OR } & \multirow{3}{*}{ IC95\% } & \multirow{3}{*}{$\mathrm{p}^{\mathrm{a}}$} \\
\hline & \multicolumn{2}{|c|}{$\mathrm{Si}$} & \multicolumn{2}{|c|}{ No } & \multicolumn{2}{|c|}{ Si } & \multicolumn{2}{|c|}{ No } & & & \\
\hline & $\%$ & $\mathrm{n}$ & $\%$ & $\mathrm{n}$ & $\%$ & $\mathrm{n}$ & $\%$ & $\mathrm{n}$ & & & \\
\hline Agresión por otros & 5,9 & 2 & 94,1 & 32 & 7,6 & 5 & 92,4 & 61 & 1,31 & 0,$24 ; 7,14$ & 0,55 \\
\hline Alergia & 8,8 & 3 & 91,2 & 31 & 13,6 & 9 & 86,4 & 57 & 1,63 & 0,$41 ; 6,47$ & 0,36 \\
\hline Intestinal y diarreica aguda & 11,7 & 4 & 88,3 & 30 & 22,7 & 15 & 77,3 & 51 & 2,21 & 0,$67 ; 7,26$ & 0,14 \\
\hline Órganos sentidos & 11,7 & 4 & 88,3 & 30 & 15,2 & 10 & 84,8 & 56 & 1,33 & 0,$38 ; 4,63$ & 0,44 \\
\hline Respiratoria aguda & 26,5 & 9 & 73,5 & 25 & 43,9 & 29 & 56,1 & 37 & 2,17 & 0,$88 ; 5,37$ & 0,08 \\
\hline Mucosas & 23,5 & 8 & 76,5 & 26 & 18,2 & 12 & 81,8 & 54 & 0,72 & 0,$26 ; 1,98$ & 0,52 \\
\hline Respiratoria & 61,8 & 21 & 38,2 & 13 & 53,0 & 35 & 47,0 & 31 & 0,69 & 0,$30 ; 1,62$ & 0,4 \\
\hline De la piel & 14,7 & 5 & 85,3 & 29 & 9,1 & 6 & 90,9 & 60 & 0,58 & 0,$16 ; 2,05$ & 0,39 \\
\hline Hernia & 0 & 0 & 100,0 & 34 & 1,5 & 1 & 98,5 & 65 & b & b & 0,47 \\
\hline Osteomuscular & 32,4 & 11 & 67,6 & 23 & 60,6 & 40 & 39,4 & 26 & 3,2 & 1,$34 ; 7,6$ & 0,007 \\
\hline Hipertensión arterial & 2,9 & 1 & 97,1 & 33 & 24,2 & 16 & 75,8 & 50 & 10,56 & 1,$33 ; 83,5$ & 0,007 \\
\hline Diabetes & 0 & 0 & 100,0 & 34 & 4,5 & 3 & 95,5 & 63 & b & b & 0,2 \\
\hline Cardiovascular & 0 & 0 & 100,0 & 34 & 12,1 & 8 & 87,9 & 58 & b & b & 0,03 \\
\hline Genito-urinaria & 0 & 0 & 100,0 & 34 & 3,1 & 2 & 96,9 & 64 & b & b & 0,3 \\
\hline Accidente tránsito & 0 & 0 & 100,0 & 34 & 3,1 & 2 & 96,9 & 64 & b & b & 0,3 \\
\hline Accidente casero & 5,9 & 2 & 94,1 & 32 & 12,1 & 8 & 87,9 & 58 & 2,21 & 0,$44 ; 11,02$ & 0,27 \\
\hline Quemadura & 5,9 & 2 & 94,1 & 32 & 4,5 & 3 & 95,5 & 63 & 0,76 & 0,$12 ; 4,79$ & 0,55 \\
\hline Hepatitis & 0 & 0 & 100,0 & 34 & 1,5 & 1 & 98,5 & 65 & b & b & 0,47 \\
\hline Dental & 0 & 0 & 100,0 & 34 & 10,6 & 7 & 89,4 & 59 & b & b & 0,04 \\
\hline Sistema nervioso & 5,9 & 2 & 94,1 & 32 & 7,6 & 5 & 92,4 & 61 & 1,3 & 0,$24 ; 7,14$ & 0,55 \\
\hline Anemia & 0 & 0 & 100,0 & 34 & 7,6 & 5 & 92,4 & 61 & b & b & 0,09 \\
\hline Estrés & 50,0 & 17 & 50,0 & 17 & 50,0 & 33 & 50,0 & 33 & 1 & 0,$43 ; 2,28$ & 1 \\
\hline Cáncer o tumor & 0 & 0 & 100,0 & 34 & 1,5 & 1 & 98,5 & 65 & b & b & 0,47 \\
\hline Picadura de animal o insecto & 44,1 & 15 & 55,9 & 19 & 22,7 & 15 & 77,3 & 51 & 0,37 & 0,$15 ; 0,90$ & 0,02 \\
\hline
\end{tabular}

a Significancia estadística con valores $p<0,05$

${ }^{\mathrm{b}}$ No se calcula dado en que una de las frecuencias observadas es 0

debido a los equipos que manejaban en el centro de aprovechamiento para la trituración de material recuperado con destino a la comercialización (Tabla 4).

Se encontraron mayores conflictos intrafamiliar y con otras personas en los recuperadores no agremiados $(p<0,05)$. Los recuperadores expresaron no ser rechazados por la sociedad por el hecho de ejercer en el reciclaje; el apoyo y reconocimiento social fue mayor en aquellos recuperadores agremiados que ejercen la labor en las organizaciones sociales.

Los recuperadores conocían las medidas de protección para enfrentar los riesgos de la labor del reciclaje. Sin embargo, este conocimiento difiere con significación estadística entre los grupos estudiados $(\mathrm{p}<0,05)$, siendo mayor en los recuperadores agremiados. El uso de las medidas de protección estuvo a favor de los recuperadores agremiados, y el cambio regular de ropa para el trabajo fue la única medida que no presentó diferencias estadísticamente significativas $(p=0,79)$. El uso de tapabocas, gafas, vestido enterizo o delantal, tapones para oídos, protector de cintura ante cargas, gorra con visera y vacunación en los últimos seis meses como medidas de prevención, presentó mayor frecuencia de uso en los recuperadores agremiados (razones de prevalencia $>1$ ).

En general, la morbilidad sentida de la población en estudio en el mes anterior priorizó eventos relacionados con molestias osteomusculares, enfermedades respiratorias y estrés (Tabla 5). No obstante, la consulta a los servicios médicos en los recuperadores fue poco frecuente $(\mathrm{p}=0,5)$.

Comparando los dos grupos de recuperadores, se encontraron diferencias estadísticamente significativas en la presentación de molestias osteomusculares, enfermedad dental, hipertensión arterial y enfermedad cardiaca: fue mayor en el grupo de los recuperadores no agremiados (Tabla 5). 
La mordedura de animal (roedores, perros, gatos, entre otros) o picadura de insecto se presentó con diferencias estadísticamente significativas entre los recuperadores ambientales, con mayor frecuencia en los recuperadores ambientales agremiados (Tabla 5).

\section{DISCUSIÓN}

El reciclaje se ha introducido en áreas rurales como una opción de empleo para sus habitantes. En este estudio, 104 personas en los cinco corregimientos de Medellín utilizaban esta actividad como una opción de ingresos para su sustento económico. El 33\% de los recuperadores estaban agremiados en organizaciones comunitarias apoyadas por entidades estatales de carácter ambiental que dedican recursos a la gestión integral de residuos en las localidades de su jurisdicción. Ribero ${ }^{\mathrm{e}}$ (2003) registró que seis de cada diez empleados en las áreas metropolitanas de Colombia trabajaban en el sector informal en el 2006 y que el 30\% de las familias dedicadas al reciclaje se encontraban asociadas a organizaciones, mientras que el $70 \%$ ejercían la labor de manera independiente. ${ }^{\mathrm{a}}$

Existe un grupo de recuperadores ambientales organizado en la zona rural de Medellín, catalogados dentro del sector de la economía informal del País, según sus características particulares.

La definición de economía informal puede tomarse tanto de la escuela estructuralista (agrupa trabajadores familiares sin remuneración, trabajadores por cuenta propia no profesionales ni técnicos, empleados del servicio doméstico, empleados y patrones de empresas del sector privado de hasta diez trabajadores) como de la escuela institucionalista, que se fundamenta en la legalidad laboral e institucional con las garantías de seguridad social en salud, afiliación a pensiones y contrato laboral. ${ }^{11}$

Resulta complejo tomar una sola definición de la informalidad en la actualidad, pues se pierde cada vez más la contratación y las prestaciones sociales en los mercados formales y se emplea fuerza asalariada a través de redes en los mercados informales. El sector informal se visualiza como un ejército de reserva del trabajo que mantiene actividades de supervivencia y se vuelven funcionales para el sistema capitalista reduciendo los costos de producción y que son manipulados por el sistema. ${ }^{11}$

Los recuperadores informales que se agremian en organizaciones sociales de base comunitaria, tales como los que se presentan en este estudio, sufren las consecuencias del estado actual de la informalidad, en tanto sus organizaciones son económicamente débiles y tienen una gran dependencia de recursos estatales locales para su continuidad en el medio.

Según Mariatti ${ }^{5}$ (2009), el trabajo que puede ser realizado en el centro comunitario, en la calle o en la casa se viste de un manto de falsa libertad fuera de la fábrica, pero profundamente determinado por el capital y por las exigencias del mercado para abaratar costos y recomponer la tasa de lucro, como ocurre con el reciclaje.

Otra mirada diferente al sector económico se ha hecho desde el sector ambiental. La creciente necesidad de la conservación de los recursos naturales ha llevado a valorar el reciclaje no sólo como una opción de ocupación para muchas personas dedicadas al aprovechamiento de los residuos sólidos, sino como una actividad productiva y de buena acogida social, dados sus diversos aportes al ambiente, a la sociedad y a la economía. ${ }^{f}$

Tal concepción del reciclaje fue también percibida por sus propios actores dentro del presente estudio. Manifestaron la importancia de su labor como una fuente de sustento económico y como aporte al sostenimiento ambiental.

Reynals (2002) $)^{\mathrm{f}}$ registra que el crecimiento de la actividad del reciclaje en las ciudades, denominado la "magnitud del fenómeno de los cartoneros", ha transformado las relaciones con estos actores sociales y ha permitido crear otras nuevas por parte de los gobiernos locales, las empresas de transporte, las organizaciones de la sociedad civil, las universidades, las organizaciones barriales, entre otras. De esa manera, se han presionado las intervenciones de los gobiernos locales, entre ellas, los proyectos de ley, contratos de concesión, promoción de la organización empresarial, concertación de políticas sociales, políticas municipales, capacitaciones y encuentros.

Medellín, en su zona rural, ha propiciado desde los entes Estatales iniciativas de organización que han permitido que se involucren personas jóvenes a la labor del reciclaje y con mejores niveles de educación, lo que ha fomentado la dignificación de la actividad.

Con la organización de la labor, según los hallazgos de este estudio, se evidencian claros beneficios para esa población, catalogada como vulnerable, tales como mejores medios e instrumentos para ejercer su labor, medidas de protección más efectivas para enfrentar los riesgos asociados, acceso a la seguridad social, reconocimiento social a la importancia de su labor, inclusión en los programas de gestión de residuos sólidos existentes en sus comunidades y el uso de medidas de protección para disminuir los riesgos.

\footnotetext{
e Ribero R, Gender Dimensions of Non - Formal Employment in Colombia (March 2003). CEDE Working Paper No. 2003-04. Disponible en: http://ssrn.com/abstract=393943 or doi:10.2139/ssrn.393943

${ }^{f}$ Reynals C. De cartoneros a recuperadores urbanos, Seminario internacional Respuestas de la sociedad civil a la emergencia social: Brasil y Argentina comparten experiencias. Sao Paulo, 2002.
} 
La diferencia entre los trabajadores del reciclaje organizados y aquellos que lo ejercen de manera independiente, en términos de condiciones óptimas de labor, fue evidente en este estudio. Esto se asemeja por lo afirmado por Mariatti ${ }^{5}$ (2009) en su análisis teórico de metamorfosis laboral y reciclaje, quien citando a Olesker $^{5}$ (2003) documenta que la informalidad del reciclaje permite salarios insuficientes, ausencia de derechos laborales y menor posibilidad de mejorar las condiciones económicas de los recuperadores.

Las edades de los recuperadores ambientales agremiados y el predominio de los hombres en la labor y del estrato socioeconómico bajo concuerdan con los estudios de Lenis et $\mathrm{al}^{4}$ (2007). Los recicladores se ubicaban principalmente en el rango de edad productiva, con mayoría en el grupo de 36 a 45 años, con prevalencia del sexo masculino y de los estratos socioeconómicos 1 y 2 . Esta distribución refleja las pocas oportunidades de empleo existentes en el país y las condiciones de pobreza a causa del desempleo, lo que los lleva a asumir ese oficio como una opción de ingresos económicos.

En este tipo de labor, las jornadas de trabajo de los recuperadores de las grandes urbes en Colombia tienden a extenderse por más de nueve horas diarias y, en promedio, se trabaja seis días durante la semana. ${ }^{7}$

Se registró predominio de trabajo de ocho horas o menos en el día y cinco días o menos durante la semana. Esta tendencia, relativamente menor a la documentada en otros estudios, puede deberse a que exista una menor cantidad de residuos en zonas rurales, puesto que tienen menor población y menor extensión de sus cabeceras en comparación con las grandes ciudades.

La similitud existente entre la morbilidad sentida por los recuperadores de ambos grupos, tanto agremiados como no agremiados, puede explicarse por las características propias del ejercicio de su labor, dado que ambos deben realizar las mismas actividades para la recuperación del material. Los recuperadores agremiados desarrollan actividades adicionales, dado que también colectan residuos biodegradables para la elaboración de compostaje, además de la preparación de algunos materiales para facilitar la comercialización.

La infección respiratoria aguda es un evento de morbilidad común en ese tipo de población, cuya relación con la labor puede darse por el desarrollo de sus actividades a la intemperie. ${ }^{7,14}$ Los problemas de espalda, los dolores musculares y el estrés se registraron como las enfermedades más prevalentes en este estudio.

La frecuencia de problemas osteomusculares ha sido reportada por otros autores en esa población. ${ }^{9}$ Se ha detectado este tipo de morbilidad, asociando factores disergonómicos de la carga de trabajo y dolor de origen musculo-esquelético, incluyendo afectaciones neuropsicologías, como efecto de la exposición a sustancias químicas, y afectaciones psicosociales relacionadas con procesos peligrosos a los cuales se exponen con su labor. ${ }^{4}$

Otros eventos de salud frecuentes son la afección intestinal y diarreica aguda, ${ }^{4}$ aunque este tipo de enfermedad no presentó prevalencia significativa en el presente estudio, lo que se debe posiblemente al desconocimiento de los recuperadores de las condiciones higiénico-sanitarias que pueden generar infecciones que desencadenan ese tipo de patología.

La prevalencia de síntomas relacionados con enfermedades entéricas y parasitismos en recuperadores ambientales es significativa en regiones con características socioeconómicas similares a las colombianas, como Manila, Egipto y localidades de Asia. A estos eventos se suman más de 35 enfermedades entre las que se destacan, además de la diarrea, la fiebre tifoidea, el cólera, la disentería, la tuberculosis, el ántrax, la poliomielitis, desordenes de piel, neumonía y malaria. ${ }^{6}$

El trabajo a la intemperie y el contacto permanente con basura puede potenciar el riesgo de accidente por mordedura de animales o picadura de insectos, que potencialmente puede transmitir enfermedades infecciosas al trabajador. Este factor es percibido por la población de recuperadores ambientales, aunque en mayor proporción por los recuperadores agremiados, posiblemente por el hecho de estar sensibilizados de las consecuencias para su salud de estos accidentes. Los recuperadores agremiados han recibido mayor número de capacitaciones en torno a los riesgos de su labor y pueden identificar las picaduras de insectos o mordeduras de animales como algo perjudicial para su salud.

Lo anterior concuerda con Acosta Cabrera et al, ${ }^{1}$ en su estudio sobre modificación de los conocimientos, actitudes y prácticas de la población en la prevención de la picada de mosquitos, en Habana, Cuba. La educación y la capacitación sobre problemas de salud permiten incrementar los conocimientos, los cambios de actitudes y la percepción de riesgo que puedan prevenir cualquier factor que incida negativamente en la salud de la población. ${ }^{1}$

Las enfermedades tropicales, emergentes y reemergentes, pueden presentarse en ese grupo de población dado su contacto constante con vectores y animales, potenciales agentes transmisores. ${ }^{9} 15$ Las condiciones de trabajo en recuperadores agremiados y no agremiados deben vigilarse y adecuarse de manera que se permita la protección de la población frente a diversos factores de riesgo en salud ocupacional y el acceso a instrumentos, equipos o herramientas que les faciliten la recolección, la preparación y el transporte del material de manera segura.

Los recuperadores ambientales tienen condiciones laborales y de salud precarias, aun agremiados en 
organizaciones comunitarias, cuya trasformación debería ser una de las prioridades del Estado, en tanto se reconocen los beneficios que trae esa actividad para la recuperación de residuos, la economía, la sociedad y la sostenibilidad ambiental.

Diferentes autores concuerdan en los beneficios de la labor ejercida por las personas dedicadas a la recuperación de residuos sólidos, evidenciando la necesidad de la inclusión del reciclaje dentro de las políticas y planes de manejo integral de residuos sólidos. ${ }^{6,17,16}$

Existe la necesidad de fomentar la organización social y empresarial de estas personas desde dimensiones culturales, políticas y económicas, con una perspectiva no sólo desde la necesidad sino desde la oportunidad para la gestión responsable de los residuos sólidos y para el aporte a la equidad en Colombia y en América Latina. ${ }^{12,13}$
La gestión social integral de residuos se convierte en una alternativa para los recuperadores ambientales que puede llegar a ser fuente de empleo estable, con condiciones laborales que les provea de herramientas e instrumentos adecuados para facilitarles la labor y protegerlos de los factores de riesgo para la salud derivados de su trabajo.

\section{AGRADECIMIENTOS}

A la Corporación Autónoma Regional del Centro de Antioquia, CORANTIOQUIA, por su gestión para la realización de esta investigación. A las organizaciones comunitarias y asesoras de recuperadores ambientales y a los recuperadores independientes de la calle, de los cinco corregimientos de la ciudad de Medellín, por su participación en este proyecto.

\section{REFERENCIAS}

1. Acosta Cabrera OS, Chalgub Moreno AM, Baydes González RO, Abraham Marcel EA. Modificación de los conocimientos, actitudes y prácticas de la población sobre la prevención de los mosquitos. Rev Cuba Hig Epidemiol. 1999;37(1):6-12.

2. Gómez JA, Agudelo A, Sarmiento J, Ronda E. Condiciones de trabajo y salud de los recicladores urbanos de Medellín (Colombia). Arch Prev Riesgos Labor. 2007;10(4):181-7.

3. Jeannot Rossi F. Desarrollo de la economía informal. Analisis Económico. 2008;23(52):133-58.

4. Lenis-Ballesteros V, Cuadros-Urrego Y, Botero S. LópezArango $Y$. Factores de riesgo biológicos en recicladores informales de la ciudad de Medellín, 2005. Rev Fac Nac Salud Publica. 2008;26(2):169-77.

5. Mariatti A. Metamorfosis laboral y reciclaje. Rev Katalysis. 2009;12(1):94-104.

6. Medina M. Scavenger cooperatives in Asia and Latin America. Resour Conserv Recycl. 2000;31(1):51-69. DOI:10.1016/S0921-3449(00)00071-9

7. Mariatti A. Metamorfosis laboral y reciclaje. Rev Katalysis. 2003;12(1):94-104.

8. Rodríguez C. En busca de alternativas económicas en tiempo de globalización: el caso de las cooperativas de recicladores de basura en Colombia. In: Santos

B, García M. Emancipación social y violencia en Colombia. Bogotá: Norma; 2004.

9. Rotaeche Montalvo V. Salud laboral versus enfermedades tropicales e importadas. Med Segur Trab. 2009;55(215):12-8. DOI:10.4321/S0465-546X2009000200002

10. Rushton L. Health hazards and waste management. $\mathrm{Br}$ Med Bull. 2003;68:183-97. DOI:10.1093/bmb/ldg034

11. Sepúlveda LA. Organización y dignificación de recicladores informales. Rev El Reto. 2003;48:23-35.

12. Sepúlveda LA. Organización del reciclaje informal en los centros urbanos: un camino para construir equidad. Rev El Reto. 2004;51:17-8.

13. Sepúlveda LA. Valoración económica del reciclaje informal. Rev El Reto. 2006;62:44-9.

14. Silveira Graudenz G. Indicadores infecciosos e inflamatórios entre trabalhadores da limpeza urbana em São Paulo. Rev Bras Saude Ocup. 2009;34(120):106-14.

15. Ullmann LS, Langoni $H$. Interactions between environment, wild animals and human leptospirosis. J Venom Anim Toxins Incl Trop Dis. 2011;17(2):119-29. DOI:10.1590/S1678-91992011000200002

16. Yepes DL, Vélez PA. Gómez WM. Factores que afectan la productividad del reciclador informal. Caso Medellín. Gest Ambient. 2008;11:85-96. 